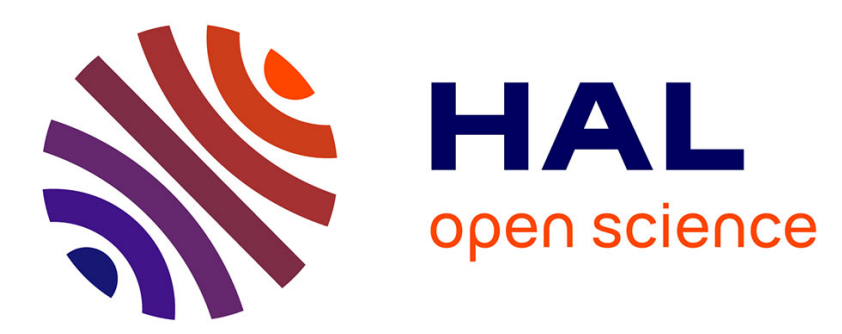

\title{
The protein tyrosine phosphatase interacting protein 51 (PTPIP51) is required for the differentiation of photoreceptors
}

Gaël Orieux, Amélie Slembrouck, Manale Bensaïd, José-Alain Sahel, Olivier Goureau

\section{To cite this version:}

Gaël Orieux, Amélie Slembrouck, Manale Bensaïd, José-Alain Sahel, Olivier Goureau. The protein tyrosine phosphatase interacting protein 51 (PTPIP51) is required for the differentiation of photoreceptors. Neuroscience, 2015, 300, pp.276-285. 10.1016/j.neuroscience.2015.05.028 • hal-01161969

\section{HAL Id: hal-01161969 \\ https://hal.sorbonne-universite.fr/hal-01161969}

Submitted on 9 Jun 2015

HAL is a multi-disciplinary open access archive for the deposit and dissemination of scientific research documents, whether they are published or not. The documents may come from teaching and research institutions in France or abroad, or from public or private research centers.
L'archive ouverte pluridisciplinaire HAL, est destinée au dépôt et à la diffusion de documents scientifiques de niveau recherche, publiés ou non, émanant des établissements d'enseignement et de recherche français ou étrangers, des laboratoires publics ou privés. 
The protein tyrosine phosphatase interacting protein 51 (PTPIP51) is required for the differentiation of photoreceptors.

Gaël Orieux, Amélie Slembrouck, Manale Bensaïd, José-Alain Sahel and Olivier Goureau

Institut de la Vision, INSERM U968 ; Sorbonne Universités, UPMC Univ Paris 06, UMR_S968 ;

CNRS UMR 7210, Paris, France

Corresponding author : Gaël Orieux

Institut de la Vision, 17 rue Moreau 75012 Paris, France

Tel : (33) 1.53.46.25.33

Fax number : (33) 153462600

Email : gael.orieux@upmc.fr 


\section{ABSTRACT}

Proliferation and differentiation of retinal progenitor cells (RPCs) are tightly controlled by extrinsic cues and distinct combinations of transcription factors leading to the generation of retinal cell type diversity. In this context, we investigated the role of the protein tyrosine phosphatase interacting protein 51 (PTPIP51) in the differentiation of RPCs. The expression pattern of PTPIP51 was analyzed by immunostaining during post-natal retinal development in the rat. Ex vivo electroporation has been used to silence or misexpress PTPIP51 in post-natal retinal explants, and the retinal phenotype was investigated after 3 to 7 days in vitro (div). PTPIP51 expression in the retina started postnatally and was maintained throughout adulthood, especially in retinal ganglion cells and in the inner segment of photoreceptor cells. Silencing of Ptpip51 expression in postnatal retina failed to modify the commitment of late RPCs in the different lineages but severely impaired the final differentiation of photoreceptors, observed by a decrease in the fraction of Rhodopsin-positive cells after 7 div. By contrast, misexpression of PTPIP51 in early or late RPCs failed to modify the differentiation of the RPCs. Our data demonstrates that PTPIP51 is implicated in the differentiation process of immature photoreceptors. Because PTPIP51 is specifically localized in the inner segment, PTPIP51 may contribute to the complex stage of maturation of the apical segment of these cells.

Keywords: retina, differentiation, photoreceptors, inner segment 


\section{INTRODUCTION}

The vertebrate retina is organized into a laminar structure comprising seven cell types, all derived from a common pool of multipotent retinal progenitor cells (RPCs) (Cayouette et al., 2006;

Bassett and Wallace, 2012; Cepko, 2014). Retinogenesis follows an evolutionarily conserved, but overlapping, sequence with retinal ganglion cells (RGCs), amacrine cells (ACs), horizontal cells (HCs) and cone photoreceptors becoming postmitotic first while rod photoreceptors, bipolar cells (BCs) and Müller glial cells (MGCs) are generated during a later stage of differentiation (Young, 1985; Rapaport et al., 2004). This sequence is under the control of both cell-extrinsic and cellintrinsic regulators (Bassett and Wallace, 2012; Boije et al., 2014; Cepko, 2014) which coordinate RPC proliferation, cell fate specification, differentiation and cell survival. Several converging lines of evidence point to specific transcription factors (homeoproteins, basic helixloop-helix, forkhead/winged helix, basic motif-leucine zipper,...) as key intrinsic regulators of retinogenesis (Furukawa et al., 1997; Hatakeyama et al., 2001; Mears et al., 2001; Dyer et al., 2003; Li et al., 2004; Matter-Sadzinski et al., 2005; Fujitani et al., 2006; Jia et al., 2009; Swaroop et al., 2010; Wang et al., 2014; Orieux et al., 2014). A number of extrinsic factors, such as the morphogen sonic hedgehog, Notch and different growth factors, have been associated with modulating RPC fate and differentiation (Austin et al., 1995; Yang, 2004; Kim et al., 2005; Wang et al., 2005; Borday et al., 2012; Mizeracka et al., 2013). Transcriptomic analysis during rat retinal development enabled us to identify a protein tyrosine phosphatase interacting protein (PTPIP) named PTPIP51 -also called FAM82A2 or RMD-3- whose mRNA expression is downregulated in post-natal retinal explants when photoreceptor differentiation is blocked (Roger et al., 2007). This protein was first identified by yeast two-hybrid screening as an interacting partner of two non-receptor protein tyrosine phosphatases, namely protein tyrosine phosphatase $1 \mathrm{~B}$ and $\mathrm{T}$ cell protein tyrosine phosphatase (Stenzinger et al., 2009b; Brobeil et al., 2011). PTPIP51 is 
expressed in diverse tissues including some regions of the mouse central nervous system (Maerker et al., 2008; Koch et al., 2009) as well as in developing and adult mammalian tissue and human carcinomas (Stenzinger et al., 2005; Koch et al., 2008). This protein has been associated to multiple cellular processes such as apoptosis (Lv et al., 2006), proliferation or migration (Yu et al., 2008a) and has also been linked with different pathological contexts such as acute myeloid leukemia (Brobeil et al., 2010) or insulin resistance (Bobrich et al., 2011). New partners of PTPIP51, such as the vesicle-associated membrane protein-associated protein B (De Vos et al., 2012; Stoica et al., 2014) and the 14-3-3 adaptor-scaffold protein (Yu et al., 2008b; Bobrich et al., 2011), have been described and their specific interaction with PTPIP51 could be involved in the regulation of calcium homeostatsis (De Vos et al., 2012), cell motility (Yu et al., 2008b) and insulin resistance (Bobrich et al., 2011).

In this study, we report a new function for PTPIP51 in retinal development during which PTPIP51 participates in regulating the differentiation of photoreceptors, more likely the final maturation rather than the cell-fate commitment.

\section{EXPERIMENTAL PROCEDURES}

\subsection{Animals.}

Timed pregnant Sprague-Dawley OFA rats were purchased from Charles River (L'Arbresle, France). The day of vaginal plug corresponded to embryonic day 0 (E0), and the day of birth corresponded to postnatal day 0 (P0). Animals were sacrificed according to the recommendations of our local ethical and animal care committee and eyes were dissected to recover neural retinal tissues (Authorization 75-865 delivered on April 302010 by the Minister of Agriculture). The methods used to secure animal tissue complied with the ARVO Statement for the Use of Animals 
in Ophthalmic and Vision Research.

\subsection{Electroporation of retinal explants.}

The central regions of the retina of embryos (E16) and newborn pups (P0) were electroporated using a CUY21 electroporator (CUY21 Single Cell BEX, Nepa Gene, Sonidel, Eire) and cultured on polycarbonate filter discs (Dutscher, Brumath, France) as previously described (Roger et al., 2006; Orieux et al., 2014).

\subsection{Cryosection and cell dissociation.}

For cryosectioning, retinal explants or dissected eyes were fixed in cold $4 \%$ paraformaldehyde (PFA) and embedded as previously described (Roger et al., 2006; Orieux et al., 2014). Cell dissociation from retinal explants was carried out as described (Roger et al., 2006).

\subsection{InSitu Hybridization}

cDNAs encoding rat PTPIP51 peptide from the position 420 to 1441 (NM_001014046) were cloned in the PCR-II-Topo vector (Life Technologies, Saint-Aubin, France). After linearization by EcoRV (Promega, Charbonnières, France), antisense riboprobes were labeled by in vitro transcription by the SP6 enzyme (Promega) with digoxigenin-11-d-UTP (Mix Digoxigenin labeling from Roche Diagnostics, Meylan, France). Tissue sections from whole eyes of P0 to P14 rats were postfixed for 10 minutes in $4 \%$ cold PFA, washed in PBS (pH 7.4), treated with proteinase $\mathrm{K}(5 \mu \mathrm{g} / \mathrm{ml}$; Promega) for 2 minutes, postfixed for 5 minutes in $4 \%$ cold PFA and washed in PBS. Slides were acetylated for 10 minutes in a $0.1 \mathrm{M}$ triethanolamine, $0.02 \mathrm{~N} \mathrm{HCl}$ and $0.25 \%$ acetic acid solution, washed in PBS $1 \%$ Triton X-100 and incubated 2 hours at room temperature (RT) with hybridization buffer $(50 \%$ formamide, $5 \times \mathrm{SSC}, 1 \times$ Denhardt's, $250 \mu \mathrm{g} / \mathrm{ml}$ yeast tRNA, and $500 \mu \mathrm{g} / \mathrm{ml}$ herring sperm DNA, $\mathrm{pH}$ 7.4) previously denatured 5 minutes at 80 ${ }^{\circ} \mathrm{C}$. Then, tissue sections were hybridized overnight at $72^{\circ} \mathrm{C}$ with riboprobes (1/200). After 
hybridization, sections were rinsed for 2 hours in $2 \times \mathrm{SSC}$ at $72^{\circ} \mathrm{C}$, and blocked in $0.1 \mathrm{M}$ Tris, $\mathrm{pH}$ 7.5, $0.15 \mathrm{M} \mathrm{NaCl}$ (B1 buffer) containing $10 \%$ normal goat serum (NGS) for 1 hour at RT. After blocking, slides were incubated overnight at RT with anti-DIG antibody conjugated with the alkaline phosphatase (1/5,000, Roche Diagnostics) in B1 buffer containing $1 \%$ NGS. After washing in B1 buffer, slides were incubated at RT for 2hours in 0.1MTris $\mathrm{pH} 9.5,0.1 \mathrm{M} \mathrm{NaCl}$, 0.1 $\mathrm{M} \mathrm{MgCl}_{2}, 0.1 \%$ Tween-20 and 0.1 M Tetramisole hydrochloride (Sigma-Aldrich, Lyon, France) (B3 buffer). The alkaline phosphatase activity was detected in B3 buffer containing nitroblue tetrazolium chloride $(337.5 \mu \mathrm{g} / \mathrm{ml})$ and 5-bromo-4-chloro-3-indolyl phosphate (175 $\mu \mathrm{g} / \mathrm{ml}$ ) (Roche Diagnostics). Sections were mounted in Eukitt mounting medium (SigmaAldrich).

\subsection{Immunostaining and image acquisition.}

Immunofluorescence staining of retinal sections or dissociated cells was performed as previously described (Orieux et al., 2014). The following primary antibodies were used: mouse anti-ATP Synthase Subunit Beta (Life Technologies); mouse anti-Brn3a (Millipore, Guyancourt, France); mouse anti-Calbindin (Swant, Marly, Switzerland); mouse anti-Calretinin (Millipore); mouse anti-GFP (Clinisciences); rabbit anti-GFP (Roche-Diagnostics); rabbit anti-CRX (gift from Dr CM Craft); mouse anti-Glutamine Synthetase (GS; Millipore); mouse anti-Go $\square$ (Millipore); rabbit anti-Otx2 (Millipore); rabbit anti-Pax6 (Millipore); rabbit anti-PKC $\alpha$ (Santa Cruz Biotechnology); rabbit anti-Recoverin (Millipore), mouse anti-Rhodopsin (R4D2, gift from Dr Molday), rabbit anti-PTPIP51 (Sigma-Aldrich). Fluorescent staining for actin was performed using Alexa Fluor-594 phalloidin (Life Technologies) and TUNEL assay was performed using the in situ cell death detection kit (Roche-Diagnostics), both completed according to the manufacturer's recommendations. Fluorescent staining signals were captured with a DM6000 
microscope (Leica Microsystems, Nanterre, France) equipped with a CCD CoolSNAPfx monochrome camera (Roper Scientific; Lisses, France) or with an Olympus FV1000 confocal microscope equipped with 405, 488 and $559 \mathrm{~nm}$ lasers. MetaMorph software (Universal Imaging Corporation; Roper Scientific) was used to measure and analyze the intensity of fluorescent staining after cell dissociation of electroplated retinal explants. The same thresholds for fluorescent staining signals were used for a given antibody combination and experiment.

\subsection{Real-time PCR (RT-PCR).}

mRNA was prepared using a Nucleospin RNA II kit (Macherey Nagel, Hoerdt, France) and cDNA synthesis was carried out using the SuperScript II Reverse Transcriptase (Life Technologies), each according to the suppliers instructions. PCR reactions were performed in a $20 \mu 1$ final volume with Power SYBR® Green PCR Master Mix (Life Technologies) and 0.25 $\mu \mathrm{M}$ primers (forward 5'GTATGCAGTGCTTTGTGGTCA3' and reverse 5'TTCGAGATGCCCTCATGCTCAG3') using the 7300 Real-Time PCR System (Life Technologies) according to the manufacturer's instructions. Gene expression threshold-cycle values were normalized to endogenous levels of S26 (rat retina) or chick Actin (DF1 cells). Samples were run in triplicate and corresponded to three independent biological samples.

\subsection{Construction of Small hairpin RNA vectors and pCIG over expression vectors.}

The pU6-Ptpip51 small hairpin RNA (shRNA) was generated by ligation of the following annealed oligonucleotides into the pGSU6 RNAi vector (Genlantis, San Diego, USA) according to the supplier's instructions: 5'-

gatccGGAGCCGACAAGACTTTCTgaagcttgAGAAAGTCTTGTCGGCTCCttttttggaagc-3' and 5'-ggccgcttccaaaaaaGGAGCCGACAAGACTTTCTcaagcttcAGAAAGTCTTGTCGGCTCCg-3'. The GeneSilencer shRNA vector contain the U6 RNA polymerase III promoter, allowing optimal 
expression of shRNA and a GFP or a RFP reporter gene under the control of CMV promoter. The ligation product was transformed into OneShot TOP10 chemically competent E.coli cells (Life Technologies). Positive clones were identified by restriction enzymes analysis of purified plasmid and verified by DNA sequencing.

The rat ptpip51 (NM_001014046) was cloned using the gateway technology (Life Technologies) into a pCIG-eGFP vector that contained an internal ribosome entry site (IRES) sequence to generate enhanced green fluorescent protein (eGFP) under the control of the same beta-actin promoter as previously described (Orieux et al., 2014). A truncated form of PTPIP51 (called PTPIP51- $\triangle \mathrm{TM}$ ), which lacks the 157 first bases, containing the transmembrane domain, was cloned in the same vector. This mutant form is called PTPIP51- $\Delta$ TM.

The different plasmids were validated by transfection of DF1 cells using FuGENE ${ }^{\circledR} 6$ Reagent (Promega) as previously described (Lelièvre et al., 2011).

\subsection{Western blot analysis.}

Protein extracts were collected in lysis buffer $\left(20 \mathrm{mM} \mathrm{NaH}_{2} \mathrm{PO}_{4}, 250 \mathrm{mM} \mathrm{NaCl}, 30 \mathrm{mM} \mathrm{NaPPi}\right.$, 0.1\% NP40, 5mM EDTA, 5mM DTT) containing protease inhibitors from transfected DF1 fibroblast cells. Homogenates were sonicated and centrifuged for $5 \mathrm{~min}$ at $5000 \mathrm{~g}$, and then $20 \mu \mathrm{g}$ of the supernatant were subjected to SDS-PAGE, as previously described (Roger et al., 2006). Antibody binding was revealed by the Enhanced Chemiluminescence System Plus (GE Healthcare) on X-Ray film.

\subsection{Quantitative analysis.}

For quantification of dissociated cells that were immunolabeled as a monolayer, a minimum of 300 and up to 1000 cells per sample were counted using MetaMorph software (Universal Imaging Corporation). TUNEL labelling was quantified by averaging the total number of positive 
cells on three different retinal explants. Quantification of marker staining is expressed as mean \pm S.E.M. Statistical analysis was performed using Prism 6 (GraphPad software; La Jolla, CA, USA) with Mann-Whitney test for all pair wise analysis. Values of $\mathrm{p}<0.05$ were considered statistically significant.

\section{RESULTS}

\subsection{PTPIP51 expression in the retina}

The temporal pattern of Ptpip51 expression was performed by RT-PCR and by in situ hybridization (ISH) during retinal development in the rat (Figure 1). RT-PCR experiments showed that the expression level increased progressively during the postnatal phase of the retinal development from birth (P0) to reach a peak at P14 before a slightly decrease in the adult retina (Figure 1A). The level of Ptpip51 mRNA was virtually undetectable before birth. ISH experiments revealed a temporal expression pattern in agreement with the qRT-PCR data with a signal at the limit of detection at $\mathrm{PO}$ and the strongest labeling obtained in retinal sections from P14 rats (Figure 1B). After P5, Ptpip51 mRNA was expressed in almost all the retinal cell layers with a weaker signal in the ganglion cell layer (GCL). Notice that Ptpip51 expression at all stages of development was principally observed in the neural retina, even though a low expression is distinguishable in the retinal pigmented epithelium, the ciliary body, the corneal epithelium and the iris. The other structures of the rat eyes, such as the sclera, the lens or the corneal stroma were virtually unlabelled (Figure 1B; P7 and P14 stages).

In order to identify retinal cell types that express PTPIP51, a series of double immunostaining experiments were performed on retinal sections at different postnatal stages of development. The expression of PTPIP51 by postnatal RGCs was demonstrated by the co-expression with Brn3a, a specific marker of RGCs (Figure 2A-A"). A weaker signal was also observed in the inner nuclear 
layer (INL), where PTPIP51 co-localized with two markers of ACs and HCs, Calbindin (ACs and HCs; Figure 2B-B") and Calretinin (ACs; Figure 2C-C"). On the other hand, PTPIP51 did not co-localized with Goo, a marker of BCs (Figure 3A-A") and neither with Glutamine Synthetase (GS), a marker of MGCs (Figure 3B-B"). Even though no immunoreactivity for PTPIP51 was observed in the outer nuclear layer (ONL), a thin PTPIP51-immunoreactive layer was visible above the ONL, in the layer corresponding to the segments of photoreceptors. Double immunostaining experiments showed that this PTPIP51-immunoreactive layer was situated just above the outer limiting membrane, revealed by the detection of the Actin using an alexa-594 coupled phalloidin (Figure 3C-C") and below the outer segment of the photoreceptors strongly immunostained by an anti-Rhodopsin antibody (Figure 3D-D”). This profile suggested that PTPIP51 could be localized in the inner segment of photoreceptors. Because this cell compartment is rich in mitochondria, we performed double immunostaining for PTPIP51 and the Beta Subunit of the ATP Synthase, that allows the identification of mitochondria (Figure 3E-E"). The co-expression of PTPIP51 and ATP Synthase confirmed that PTPIP51 localization in the outer part of the retina was restricted in the photoreceptor inner segments.

\subsection{The differentiation of photoreceptor precursors is altered in absence of PTPIP51}

In order to evaluate the function of PTPIP51 during the postnatal development of the retina, we knocked down PTPIP51 expression by shRNA-interference in rat retinal explants. To inhibit PTPIP51 expression, we utilized a pU6-based shRNA system co-expressing a GFP reporter gene in order to follow the electroporated cells (details in Materials and Methods). The efficiency of silencing was first evaluated in DF1 cells overexpressing PTPIP51 after transfection with pCIGPTPIP51-eGFP (Figure 4A). Cotransfection with vector silencing Ptpip51 (pU6-shPTPIP51RFP) prevented the expression of Ptpip51 mRNA compared to control cells co-transfected with 
pU6-shLuciferase-RFP (Figure 4Aa). Double-positive cells for GFP and RFP observed in control conditions (Figure 4Ab) where never detected after co-transfection with pU6-shPTPIP51-RFP vector (Figure 4Ac). Because PTPIP51 is not expressed before birth in the rat retina, we electroporated explants from P0 retina and focused our attention on the late-born retinal cells, i.e. the rod photoreceptors, the MGCs and BCs (Figure 4B-G). Three days after electroporation, the percentage of photoreceptor precursors immunoreactive for Crx was similar in that observed in shPTPIP51-electroporated explants $(89.26 \pm 1.1 \% ; n=4)$ compared to shLuciferaseelectroporated control explants $(88.68 \pm 1.6 \% ; n=4)($ Figure $4 B)$. These results indicate that three days after silencing PTPIP51 expression in P0 retinal explants, the commitment of the RPCs into the photoreceptor lineage was not modified. As expected at this stage, the residual RPCs normally committed into the Pax6 lineage (ACs and HCs) were not affected at all by down-regulation of PTPIP51 (11.38 $\pm 1.27 \%$ after PTPIP51 silencing versus $11.56 \pm 1.69 \%$ on the control; $n=4)$. Because PTPIP51 is expressed in the inner segments of the photoreceptors (Figure 3), we questioned whether the PTPIP51 silencing could influence the maturation of the photoreceptors. Three days after electroporation, a downward trend in the proportion of more mature photoreceptors expressing Recoverin or Rhodopsin was observed in shPTPIP51electroporated explants (Figure 4B). Seven days after electroporation, no significant changes were observed in the proportion of Crx-positive photoreceptors following PTPIP51 silencing (Figure 4C). However, specific evaluation of more differentiated photoreceptors, corresponding to the number of cells expressing Rhodopsin and Recoverin among the GFP-positive population, revealed a greater decrease of immunopositive cells in shPTPIP51-electroporated explants (10.23 $\pm 2.03 \%$ and $14.51 \pm 1.20 \%$ respectively; $n=4$ ) compared to shLuciferase-electroporated explants $(64.18 \pm 2.17 \%$ and $51.53 \pm 4.88 \%$ respectively; $n=4)($ Figure $4 C)$. Figures $4 \mathrm{D}$ and 4E illustrate the decrease of electroporated cells (GFP-positive) immunopositive for Rhodopsin in 
shPTPIP51-electroporated explants (Figure 4E) compared to the control (Figure 4D). Evaluation of the proportion of BCs and MGCs identified with Go $\alpha$ and GS respectively revealed either no significant changes following PTPIP51 silencing (Figure 4C). To exclude cell death as a cause of the large decrease of photoreceptors in shPTPIP51-electroporated explants, we performed TUNEL staining to identify apoptotic cells. Figures $4 \mathrm{~F}$ and $4 \mathrm{G}$ show that the majority of positive cells was observed in the GCL, in both shPTPIP51-electroporated and shLuciferase-

electroporated explants three days after electroporation. This observation was probably due to the retrograde degeneration of the RGCs after the optic nerve section and the absence of projection targets in vitro. Besides this expected wave of cell death in the GCL, the number of TUNELpositive cells among the GFP-positive population was very low in both shPTPIP51electroporated $(2.1 \pm 0.3$ cells per section; Figure $4 \mathrm{G})$ and shLuciferase-electroporated explants $(1.8 \pm 0.2$ cells per section; Figure $4 \mathrm{~F})$, suggesting that rare apoptosis of differentiating photoreceptors could not be responsible for the large decreased rate of photoreceptors after PTPIP51 silencing.

\subsection{Misexpression of PTPIP51 do not influence the retinal development}

In order to define the role of PTPIP51 during the development of the retina, we performed misexpression experiments on P0 retinal explants. . Western-blot analysis of transfected DF1 cells with pCIG constructs (pCIG-PTPIP51-eGFP and pCIG-PTPIP51-ATM-eGFP) confirmed the expression of the full-length form of PTPIP51 or a truncated form (PTPIP51- $\triangle \mathrm{TM}$ ) lacking the transmembrane domain (Figure 5Aa). In E16 electroporated retinal explants, where no endogenous expression of PTPIP51 has been detected, PTPIP51 immunoreactivity is observed after electroporation with pCIG-PTPIP51-eGFP in GFP-positive cells only (Figure 5Ac). P0 
retinal explants were then electroporated with the full-length form of PTPIP51 or the truncated form (PTPIP51- $\triangle \mathrm{TM}$ ). After nine days in vitro, we did not observed any modification in the proportion of the different late born retinal cells derived from the electroporated RPCs at P0 (Figure 5B). The number of Rhodopsin, Recoverin, Crx, or Otx2-positive photoreceptor cells among the GFP-positive population showed no difference between pCIG-eGFP and pCIGPTPIP51 electroporated explants. The ratio of BCs (PKC -positive cells) and MGCs (GS-positive cells) remained also unchanged after PTPIP51 misexpression (Figure 5B).

We next examined whether PTPIP51 overexpression in E16 RPCs that do not normally express this protein could affect their cell fate and differentiation. Seven days after electroporation, quantification of the proportion of ACs and HCs identified with Pax6 and Calretinin revealed no significant changes following PTPIP51 overexpression(Figure 5C). In the same way, the rate of photoreceptors immunoreactive for Rhodospsin, Recoverin of Crx was not modified in PTPIP51electroporated explants (Figure 5C).

Since PTPIP51 overexpression has been reported to induce cell death in vitro (Lv et al. 2006), and in order to exclude the fact that the absence of effect after PTPIP51 overexpression could be due to a massive cell death of PTPIP51-overexpressing cells, we evaluated the cell death in retinal explants three days after electroporation. An important number of apoptotic cells was observed in the GCL of retinal explants electroporated with PTPIP51 (Figure 5D), but the number of TUNEL-positive cells in the ONL within the GFP-positive population was very low $(2.8 \pm 0.4$ cells per section). A very similar number of apoptotic cells were observed $(2.3 \pm 0.5$ cells per section) in retinal explants electroporated with PTPIP51- $\Delta$ TM (Figure 5D), previously described as unable to induce cell death (Lv et al., 2006). These results demonstrated that overexpression of PTPIP51 in the RPCs is not pro-apoptotic. 


\section{DISCUSSION}

In the present study, we reported the expression of PTPIP51 in the rat retina during post-natal development, specifically in RGCs, and in photoreceptor cells in more mature retina as previously suggested in mouse (Maerker et al., 2008) . Furthermore, we have also demonstrated that PTPIP51 expression in the photoreceptor cells is specifically localized at the level of the inner segments. Lastly, we reported the expression of PTPIP51 in the inner nuclear layer specifically in HCs and ACs for the first time. However, unlike Maerker et al. (2008) we did not detect significant expression of Ptpip51 mRNA in the retina before birth. The difference might originate from the methodology used, because Maerker et al. (2008) seem to have used whole eye's extracts for their PCR and western blotting experiments. Since their study clearly reported a PTPIP51 expression in other ocular tissues such as the cornea or the lens, it's difficult to affirm that embryonic retina expressed PTPIP51. In our RT-qPCR study, we have specifically extracted the RNA from isolated retina at each stage of development and confirm that Ptpip51 mRNA was not detected before birth (Figure 1A). Another explanation could be related to the species studied, namely rat or mice.

Interestingly, the subcellular localization noticeably differed in the different retinal cell types. The expression of PTPIP51 was restricted to the cytoplasm of ACs and HCs, while PTPIP51 was found to be also expressed in the nucleus of the RGCs. Regarding the photoreceptor inner segments, the co-localization of PTPIP51 with the ATP-synthase, a mitochondrial marker, suggests the association of PTPIP51 with this organelle, as previously described in other cell types (Lv et al., 2006; De Vos et al., 2012; Stoica et al., 2014). The variability of the subcellular localization in the rat retina is in agreement with previous studies, which reported different localization for PTPIP51 according to the cell type, but also resulting from the phosphorylation 
status of PTPIP51 and from the type of the interacting proteins involved (Stenzinger et al., 2009b; Brobeil et al., 2010, 2012; Bobrich et al., 2013). Furthermore, different isoforms of variable molecular weight have been observed in many tissues, including mouse brain and retina (Stenzinger et al., 2005, 2009a; Maerker et al., 2008; Koch et al., 2009; Brobeil et al., 2010). Experimental finding were limited to Western blot analyses but predictive alternative splicing from bioinformatics analysis of the coding ORF of PTPIP51 (Brobeil et al., 2011) could explain the generation of different PTPIP51 isoforms. Moreover, the N-terminal putative transmembrane motif has been described to be required for the localization of PTPIP51 at the membrane level (Lv et al., 2006), suggesting that predictive isoforms devoid of this motif may exhibit a different localization from the full-length isoform (Stenzinger et al., 2009b). All these data, to be experimentally confirmed, could also explain the variability in the localization of PTPIP51 found in the retina.

Our study demonstrated that PTPIP51 plays an important role during the last phase of the retinal development and is required for full differentiation of photoreceptors since the generation of Rhodopsin-positive cells in P0 retinal explants is largely reduced in absence of PTPIP51. Our data suggest that PTPIP51 is not involved in the commitment of RPCs towards the photoreceptor cell fate but more specifically implicated in the maturation of the photoreceptor precursors in fully-differentiated cells. Indeed, the silencing of PTPIP51 expression did not affect the generation of the CRX-positive photoreceptor precursors. This is in agreement with the downregulation of PTPIP51 expression in P0 retinal explants treated with ciliary neurotrophic factor (Roger et al., 2007), where an inhibition of the rod photoreceptor differentiation was observed (Ezzeddine et al., 1997; Schulz-Key et al., 2002; Rhee et al., 2004; Rhee and Yang, 2010). The PTPIP51 protein also known as regulator of microtubule dynamics protein 3 (RMD-3) belongs to a family of microtubule-associated proteins and its interaction with microtubular 
cytoskeleton has been recently demonstrated by duolink proximity ligation assay (Brobeil et al., 2012). A potential role of PTPIP51 in cell morphology and motility has been postulated in different cell lines via its interaction with the 14-3-3 adaptor-scaffold protein (Yu et al., 2008b). Moreover, PTPIP51 has been reported to be expressed in different ciliated epithelia such as the brain ependymal cells, the respiratory epithelium, the uterine epithelium or efferent ductules of the testis (Stenzinger et al., 2005, 2009a), where PTPIP51 protein appeared to be localized within the motile cilium, close to the microtubules and the basal body (Stenzinger et al., 2005).

Considering that the outer segment of the photoreceptor is a modified cilium (Adams et al., 2007; Kennedy and Malicki, 2009; Pearring et al., 2013; Guemez-Gamboa et al., 2014), it might be possible that PTPIP51 participates in the regulation of the dynamics of the connecting cilium or the intraflagellar transport machinery, occurring during both the outer segment morphogenesis and renewing.

In conclusion, our results have shown that PTPIP51 expressed in post-natal retina contributes to the final differentiation of photoreceptors, probably by participating in the complex formation of the photoreceptor segments.

\section{ACKNOWLEDGEMENTS.}

We thank Dr C. Craft and Dr R. Molday for antibodies. We acknowledge S. Thomasseau for her technical assistance. We are grateful to S. Fouquet of the « Institut de la Vision » Imaging Facility, Dr M. Garita for critical reading. This work was financed by INSERM, UPMC Université Paris 6 and Retina France association. This work was performed in the frame of the LABEX LIFESENSES [ANR-10-LABX-65] and was supported by French state funds managed by the ANR within the Investissements d'Avenir programme [ANR-11-IDEX-0004-02]. The « Institut de la Vision » imaging facility was supported by "Departement de Paris" and by "Region 
Ile de France”. A. Slembrouck was supported by the ANR [GPiPS: ANR-2010-RFCS005].

\section{REFERENCES}

Adams NA, Awadein A, Toma HS (2007) The retinal ciliopathies. Ophthalmic Genet 28:113125.

Austin CP, Feldman DE, Ida J a, Cepko CL (1995) Vertebrate retinal ganglion cells are selected from competent progenitors by the action of Notch. Development 121:3637-3650.

Bassett EA, Wallace VA (2012) Cell fate determination in the vertebrate retina. Trends Neurosci 35:565-573.

Bobrich M, Brobeil A, Mooren FC, Krüger K, Steger K, Tag C, Wimmer M (2011) PTPIP51 interaction with PTP1B and 14-3-3 $\beta$ in adipose tissue of insulin-resistant mice. Int J Obes (Lond) 35:1385-1394.

Bobrich MA, Schwabe SA, Brobeil A, Viard M, Kamm M, Mooren FC, Krüger K, Tag C, Wimmer M (2013) PTPIP51: a new interaction partner of the insulin receptor and PKA in adipose tissue. J Obes 2013:476240.

Boije H, MacDonald RB, Harris WA (2014) Reconciling competence and transcriptional hierarchies with stochasticity in retinal lineages. Curr Opin Neurobiol 27:68-74.

Borday C, Cabochette P, Parain K, Mazurier N, Janssens S, Tran HT, Sekkali B, Bronchain O, Vleminckx K, Locker M, Perron M (2012) Antagonistic cross-regulation between Wnt and Hedgehog signalling pathways controls post-embryonic retinal proliferation. Development 139:3499-3509.

Brobeil A, Bobrich M, Wimmer M (2011) Protein tyrosine phosphatase interacting protein 51--a jack-of-all-trades protein. Cell Tissue Res 344:189-205.

Brobeil A, Graf M, Eiber M, Wimmer M (2012) Interaction of PTPIP51 with Tubulin, CGI-99 and Nuf2 During Cell Cycle Progression. Biomolecules 2:122-142.

Brobeil A, Graf M, Oeschger S, Steger K, Wimmer M (2010) PTPIP51-a myeloid lineage specific protein interacts with PTP1B in neutrophil granulocytes. Blood Cells Mol Dis 45:159-168.

Cayouette M, Poggi L, Harris WA (2006) Lineage in the vertebrate retina. Trends Neurosci 29:563-570. 
Cepko C (2014) Intrinsically different retinal progenitor cells produce specific types of progeny. Nat Rev Neurosci 15:615-627.

De Vos KJ, Mórotz GM, Stoica R, Tudor EL, Lau K-F, Ackerley S, Warley A, Shaw CE, Miller CCJ (2012) VAPB interacts with the mitochondrial protein PTPIP51 to regulate calcium homeostasis. Hum Mol Genet 21:1299-1311.

Dyer MA, Livesey FJ, Cepko CL, Oliver G (2003) Prox1 function controls progenitor cell proliferation and horizontal cell genesis in the mammalian retina. Nat Genet 34:53-58.

Ezzeddine ZD, Yang X, DeChiara T, Yancopoulos G, Cepko CL (1997) Postmitotic cells fated to become rod photoreceptors can be respecified by CNTF treatment of the retina.

Development 124:1055-1067.

Fujitani Y, Fujitani S, Luo H, Qiu F, Burlison J, Long Q, Kawaguchi Y, Edlund H, MacDonald RJ, Furukawa T, Fujikado T, Magnuson M, Xiang M, Wright CVE (2006) Ptf1a determines horizontal and amacrine cell fates during mouse retinal development. Development 133:4439-4450.

Furukawa T, Morrow EM, Cepko CL (1997) Crx, a novel otx-like homeobox gene, shows photoreceptor-specific expression and regulates photoreceptor differentiation. Cell 91:531541.

Guemez-Gamboa A, Coufal NG, Gleeson JG (2014) Primary Cilia in the Developing and Mature Brain. Neuron 82:511-521.

Hatakeyama J, Tomita K, Inoue T, Kageyama R (2001) Roles of homeobox and bHLH genes in specification of a retinal cell type. Development 128:1313-1322.

Jia L, Oh EC, Ng L, Srinivas M, Brooks M, Swaroop A, Forrest D (2009) Retinoid-related orphan nuclear receptor RORbeta is an early-acting factor in rod photoreceptor development. Proc Natl Acad Sci U S A 106:17534-17539.

Kennedy B, Malicki J (2009) What drives cell morphogenesis: a look inside the vertebrate photoreceptor. Dev Dyn 238:2115-2138.

Kim J, Wu H-H, Lander AD, Lyons KM, Matzuk MM, Calof AL (2005) GDF11 controls the timing of progenitor cell competence in developing retina. Science 308:1927-1930.

Koch P, Stenzinger a, Viard M, Märker D, Mayser P, Nilles M, Schreiner D, Steger K, Wimmer M (2008) The novel protein PTPIP51 is expressed in human keratinocyte carcinomas and their surrounding stroma. J Cell Mol Med 12:2083-2095.

Koch P, Viard M, Stenzinger A, Brobeil A, Tag C, Steger K, Wimmer M (2009) Expression profile of PTPIP51 in mouse brain. J Comp Neurol 517:892-905. 
Lelièvre EC, Lek M, Boije H, Houille-Vernes L, Brajeul V, Slembrouck A, Roger JE, Sahel JA, Matter JM, Sennlaub F, Hallböök F, Goureau O, Guillonneau X (2011) Ptf1a/Rbpj complex inhibits ganglion cell fate and drives the specification of all horizontal cell subtypes in the chick retina. Dev Biol 358:296-308.

Li S, Mo Z, Yang X, Price SM, Shen MM, Xiang M (2004) Foxn4 controls the genesis of amacrine and horizontal cells by retinal progenitors. Neuron 43:795-807.

Lv BF, Yu CF, Chen YY, Lu Y, Guo JH, Song QS, Ma DL, Shi TP, Wang L (2006) Protein tyrosine phosphatase interacting protein 51 (PTPIP51) is a novel mitochondria protein with an N-terminal mitochondrial targeting sequence and induces apoptosis. Apoptosis 11:14891501.

Maerker D, Stenzinger A, Schreiner D, Tag C, Wimmer M (2008) Expression of PTPIP51 during mouse eye development. Histochem Cell Biol 129:345-356.

Matter-Sadzinski L, Puzianowska-Kuznicka M, Hernandez J, Ballivet M, Matter J-M (2005) A bHLH transcriptional network regulating the specification of retinal ganglion cells. Development 132:3907-3921.

Mears AJ, Kondo M, Swain PK, Takada Y, Bush RA, Saunders TL, Sieving PA, Swaroop A (2001) Nrl is required for rod photoreceptor development. Nat Genet 29:447-452.

Mizeracka K, Demaso CR, Cepko CL (2013) Notch1 is required in newly postmitotic cells to inhibit the rod photoreceptor fate. Development 140:3188-3197.

Orieux G, Picault L, Slembrouck A, Roger JE, Guillonneau X, Sahel J-A, Saule S, McPherson JP, Goureau O (2014) Involvement of Bcl-2-associated transcription factor 1 in the differentiation of early-born retinal cells. J Neurosci 34:1530-1541.

Pearring JN, Salinas RY, Baker SA, Arshavsky VY (2013) Protein sorting, targeting and trafficking in photoreceptor cells. Prog Retin Eye Res 36:24-51.

Rapaport DH, Wong LL, Wood ED, Yasumura D, LaVail MM (2004) Timing and topography of cell genesis in the rat retina. J Comp Neurol 474:304-324.

Rhee K-D, Goureau O, Chen S, Yang X-J (2004) Cytokine-induced activation of signal transducer and activator of transcription in photoreceptor precursors regulates rod differentiation in the developing mouse retina. J Neurosci 24:9779-9788.

Rhee K-D, Yang X-J (2010) Function and mechanism of CNTF/LIF signaling in retinogenesis. Adv Exp Med Biol 664:647-654.

Roger J, Brajeul V, Thomasseau S, Hienola A, Sahel J-A, Guillonneau X, Goureau O (2006) Involvement of Pleiotrophin in CNTF-mediated differentiation of the late retinal progenitor cells. Dev Biol 298:527-539. 
Roger J, Goureau O, Sahel J-A, Guillonneau X (2007) Use of suppression subtractive hybridization to identify genes regulated by ciliary neurotrophic factor in postnatal retinal explants. Mol Vis 13:206-219.

Schulz-Key S, Hofmann H-D, Beisenherz-Huss C, Barbisch C, Kirsch M (2002) Ciliary neurotrophic factor as a transient negative regulator of rod development in rat retina. Invest Ophthalmol Vis Sci 43:3099-3108.

Stenzinger A, Kajosch T, Tag C, Porsche A, Welte I, Hofer HW, Steger K, Wimmer M (2005) The novel protein PTPIP51 exhibits tissue- and cell-specific expression. Histochem Cell Biol 123:19-28.

Stenzinger A, Märker D, Koch P, Hoffmann J, Baal N, Steger K, Wimmer M (2009a) Protein tyrosine phosphatase interacting protein 51 (PTPIP51) mRNA expression and localization and its in vitro interacting partner protein tyrosine phosphatase $1 \mathrm{~B}$ (PTP1B) in human placenta of the first, second, and third trimester. J Histochem Cytochem 57:143-153.

Stenzinger A, Schreiner D, Koch P, Hofer H, Wimmer M (2009b) Cell and Molecular Biology of the Novel Protein Tyrosine- Phosphatase-Interacting Protein 51. Int Rev Cell Mol Biol 275:183-246.

Stoica R, De Vos KJ, Paillusson S, Mueller S, Sancho RM, Lau K-F, Vizcay-Barrena G, Lin WL, Xu Y-F, Lewis J, Dickson DW, Petrucelli L, Mitchell JC, Shaw CE, Miller CCJ (2014) ER-mitochondria associations are regulated by the VAPB-PTPIP51 interaction and are disrupted by ALS/FTD-associated TDP-43. Nat Commun 5:3996.

Swaroop A, Kim D, Forrest D (2010) Transcriptional regulation of photoreceptor development and homeostasis in the mammalian retina. Nat Rev Neurosci 11:563-576.

Wang S, Sengel C, Emerson MM, Cepko CL (2014) A gene regulatory network controls the binary fate decision of rod and bipolar cells in the vertebrate retina. Dev Cell 30:513-527.

Wang Y, Dakubo GD, Thurig S, Mazerolle CJ, Wallace V (2005) Retinal ganglion cell-derived sonic hedgehog locally controls proliferation and the timing of RGC development in the embryonic mouse retina. Development 132:5103-5113.

Yang X-J (2004) Roles of cell-extrinsic growth factors in vertebrate eye pattern formation and retinogenesis. Semin Cell Dev Biol 15:91-103.

Young RW (1985) Cell differentiation in the retina of the mouse. Anat Rec 212:199-205.

Yu C, Han W, Shi T, Lv B, He Q, Zhang Y, Li T, Zhang Y, Song Q, Wang L, Ma D (2008a) PTPIP51, a novel 14-3-3 binding protein, regulates cell morphology and motility via RafERK pathway. Cell Signal 20:2208-2220. 
Yu C, Han W, Shi T, Lv B, He Q, Zhang Y, Li T, Zhang Y, Song Q, Wang L, Ma D (2008b) PTPIP51, a novel 14-3-3 binding protein, regulates cell morphology and motility via RafERK pathway. Cell Signal 20:2208-2220. 


\section{FIGURE LEGENDS}

Figure 1. Expression pattern of Ptpip51 in the developing mouse retina.

$\boldsymbol{A}$, RT-PCR on mRNA extracts from E16 to P14 rat retina. Relative expression is normalized to retina at P0. ND (Not Detected). $\boldsymbol{B}$, mRNA ISH of Ptpip51 in retinal section from P0 to P14 rats. Image acquisitions were obtained from the central retina excepted for the P7 stage in order to illustrate the lower expression or the absence of labeling in other ocular tissue like the ciliary body. c: cornea; cb: ciliary body; gcl: ganglion cell layer; i: iris; inl: inner nuclear layer; l: lens; onl: outer nuclear layer; r: retina; sc: sclera. Scale bars: $50 \mu \mathrm{m}$.

\section{Figure 2. Expression of PTPIP51 in early-born retinal cells.}

Sections of central retina close to the optic nerve at P10 were immunostained for PTPIP51 (green) and different cell-type markers (red). Small panels display the distribution of labelled cells for PTPIP51 $(\boldsymbol{A}, \boldsymbol{B}, \boldsymbol{C}), \operatorname{Brn} 3 \mathrm{a}\left(\boldsymbol{A}^{\prime}\right)$, Calbindin $\left(\boldsymbol{B}^{\prime}\right)$ and Calretinin $\left(\boldsymbol{C}^{\prime}\right)$ in the tissue visualized

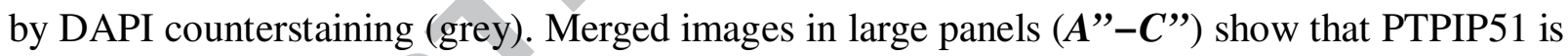
expressed in Brn3a-positive RGCs ( $\boldsymbol{A}$ ”; arrows), Calbindin-positive HCs (B”; arrows), Calbindin-positive ACs ( $\boldsymbol{B}$ ” ; arrowheads), Calretinin-positive ACs ( $\boldsymbol{C}$ ” ; arrows) and Calretininpositive displaced-ACs (C”; arrowheads). Scale bars: $30 \mu \mathrm{m}$ (small panels) or $15 \mu \mathrm{m}$ (large panels).

Figure 3. Expression of PTPIP51 in late-born cells is restricted to photoreceptor cells. Sections of central retina close to the optic nerve at P14 were immunostained for PTPIP51 (green) and different cell-type markers (red). Small panels display the distribution of labeled cells 


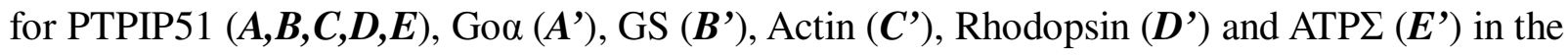
tissue is visualized through DAPI counterstaining (grey). Merged images $(\boldsymbol{A}$ ”- $\boldsymbol{E}$ ”) show that PTPIP51 is not expressed in Go $\alpha$-positive BCs (A’) or GS-positive MGCs $(\boldsymbol{B}$ ”). PTPIP51 was neither detected in the outer nuclear layer $(\boldsymbol{C}-\boldsymbol{E})$, nor in the Rhodopsin-stained rod outer segments $\left(\boldsymbol{D}^{\text {”) }}\right.$ but was present just above the outer limiting membrane revealed by the phalloidin-labelled actin $(\boldsymbol{C}$ ') and colocalized with the mitochondrial marker ATP-synthase $(\mathrm{ATP} \Sigma)$, mainly located in the photoreceptor inner segments $(\boldsymbol{E}$ ”). Dotted line delimitated areas indicate the location of each corresponding enlargement. Scale bars: $30 \mu \mathrm{m}$ (small panels) or 10 $\mu \mathrm{m}$ (large panels).

\section{Figure 4. Ptpip51 silencing in late RPCs disrupts the differentiation of rod-photoreceptors.}

$\boldsymbol{A}$, Validation of shRNA silencing in cultured DF1 cells co-transfected with pCIG-eGFPPTPIP51 and pU6-shPTPIP51-RFP vectors. (a) Q-PCR on mRNA extracts confirmed that shRNA against Ptpip51 (pU6-shPTPIP51-RFP) largely decreased Ptpip51 expression (pCIGPTPIP51-eGFP) compared to control (pU6-shLuciferase-RFP); Data were normalized to DF1 cells transfected with control pCIG-eGFP (pCIG_Ø). (b,c) Fluorescence staining confirmed that GFP/RFP-double-positive cells (arrowheads) are visible when pCIG-PTPIP51-eGFP was cotransfected with pU6-shLuciferase-RFP (control) vectors (b) but are missing after co-transfection with pU6-shPTPIP51-RFP (c). B-G, Retinal explants from P0 pups were electroporated with either pU6-shPTPIP51-eGFP or pU6-shLuciferase-eGFP (control) vectors. After 3 or 7 div, dissociated cells from retinal explants were co-immunostained for GFP with markers of different retinal cell subtypes $(\boldsymbol{B}-\boldsymbol{E})$. The graphs show the ratio of specific marker-positive cells from the GFP-positive cell population after $3(\boldsymbol{B})$ or $7 \mathrm{div}(\boldsymbol{C})$. No change was observed in the cell 
populations analysed 3 div after Ptpip51 silencing (B). After 7 div, silencing Ptpip51 led to a 84 $\%$ and $72 \%$ drop in positive cells for Rhodopsin (Rhod) and Recoverin (Recov) respectively $(\boldsymbol{C})$. Conversely, the number of Goa-positive BCs and GS-positive MGCs were not affected by silencing Ptpip51 (C). Immunohistochemistry with antibody against Rhodopsin revealed a large decrease in the ratio of Rhodopsin-positive cells from the pU6-shPTPIP51-eGFP electroporated population $(\boldsymbol{E})$ compared to the control electroporated with pU6-shLuciferase-eGFP $(\boldsymbol{D})$. Arrows arrowheads indicate the Rhodopsin-negative / GFP-positive and the Rhodopsin-positive / GFPpositive cells respectively. After 3 div, frozen sections from retinal explants were immunostained for GFP and processed for cell death evaluation using TUNEL-labelling $(\boldsymbol{F}, \boldsymbol{G})$. Retinal cells undergoing apoptosis were TUNEL-labeled (red) in retinal sections. Rare double- GFP/TUNEL positive cells (arrow) were detected in both control or pU6-shPTPIP51-eGFP electroporated explants. Calret: calretinin; Rhod: Rhodopsin; Recov: Recoverin. Values represent the mean \pm SEM (*: p<0.05; **: p<0.01) from at least four experiments. Scale bar: $30 \mu \mathrm{m}(\boldsymbol{A}, \boldsymbol{D}, \boldsymbol{E})$ or 50 $\mu \mathrm{m}(\boldsymbol{F}, \mathbf{G})$.

\section{Figure 5. Ectopic expression of PTPIP51 in retinal explants did not affect the retinal}

\section{development.}

$\boldsymbol{A}$, Validation of PTPIP51 expression cloned into pCIG-eGFP vector in transfected DF1 cells (a, b) and in electroporated retinal explants (c). Western-blotting analysis using DF1 cell lysates confirmed the production of the full form of PTPIP51 and its mutant PTPIP51- $\triangle \mathrm{TM}(\mathbf{a})$; molecular weight are indicated (in $\mathrm{kDa}$ ) and actin was used as a loading control (b). Coimmunostaining of GFP (green) and PTPIP51 (red) on frozen sections from E16 retinal explants, 3 div after electroporation, confirmed the expression of PTPIP51 in or GFP-positive 
electroporated cells $(\boldsymbol{c}) . \boldsymbol{B}-\boldsymbol{D}$, Retinal explants from E16 embryos and P0 pups were electroporated with pCIG-PTPIP51-eGFP, pCIG-PTPIP51-ATM-eGFP or control vector pCIGeGFP (pCIG- $\varnothing)$. Dissociated cells from P0+9 div $(\boldsymbol{B})$ or E16+7 div $(\boldsymbol{C})$ retinal explants were coimmunostained for GFP and markers of different retinal cell subtypes. The graph shows the ratio of specific marker-positive cells from the GFP-positive cell population. Forced expression of PTPIP51 or the truncated form, PTPIP51- $\triangle \mathrm{TM}$, did not modify the differentiation of early $(\boldsymbol{C})$ or late RPCs (B) compared to the control. Calret: calretinin; Rhod: Rhodopsin; Recov: Recoverin. $\boldsymbol{D}$, After 3 div, cells undergoing apoptosis were TUNEL-labeled in retinal sections. Majority of TUNEL-postive cells (red) were located in the ganglion cell layer and were GFP-negative regardless of the electroporated vector. Scale bars: $30 \mu \mathrm{m}(\boldsymbol{A})$ or $50 \mu \mathrm{m}(\boldsymbol{D})$. 
Figure 1

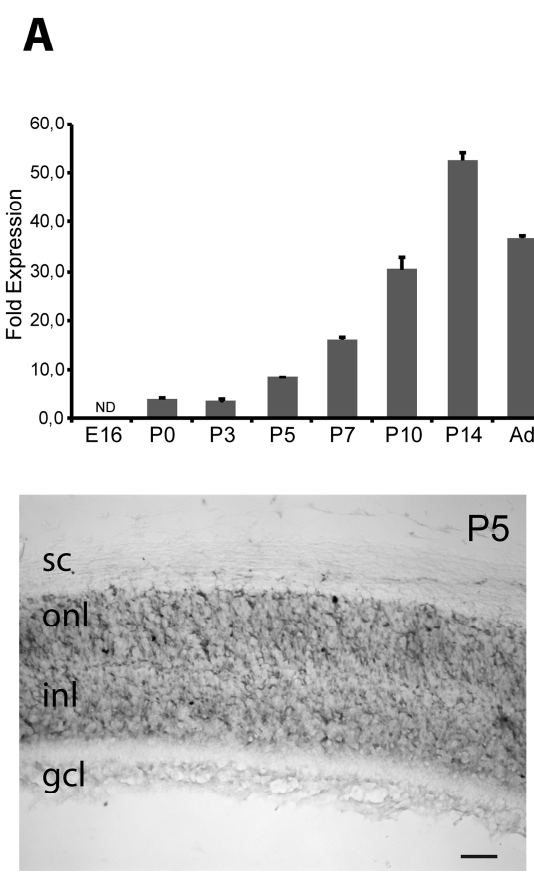

B

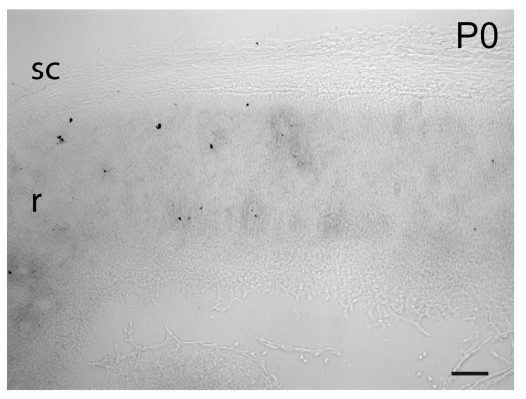

P7
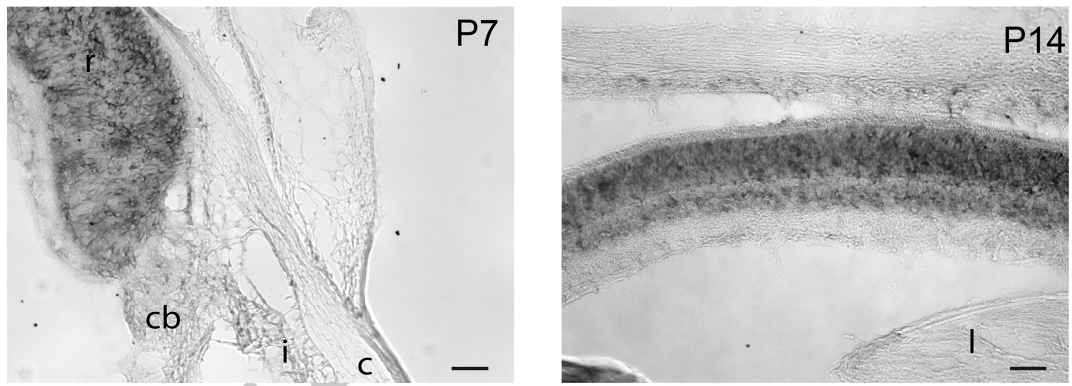
Figure 2
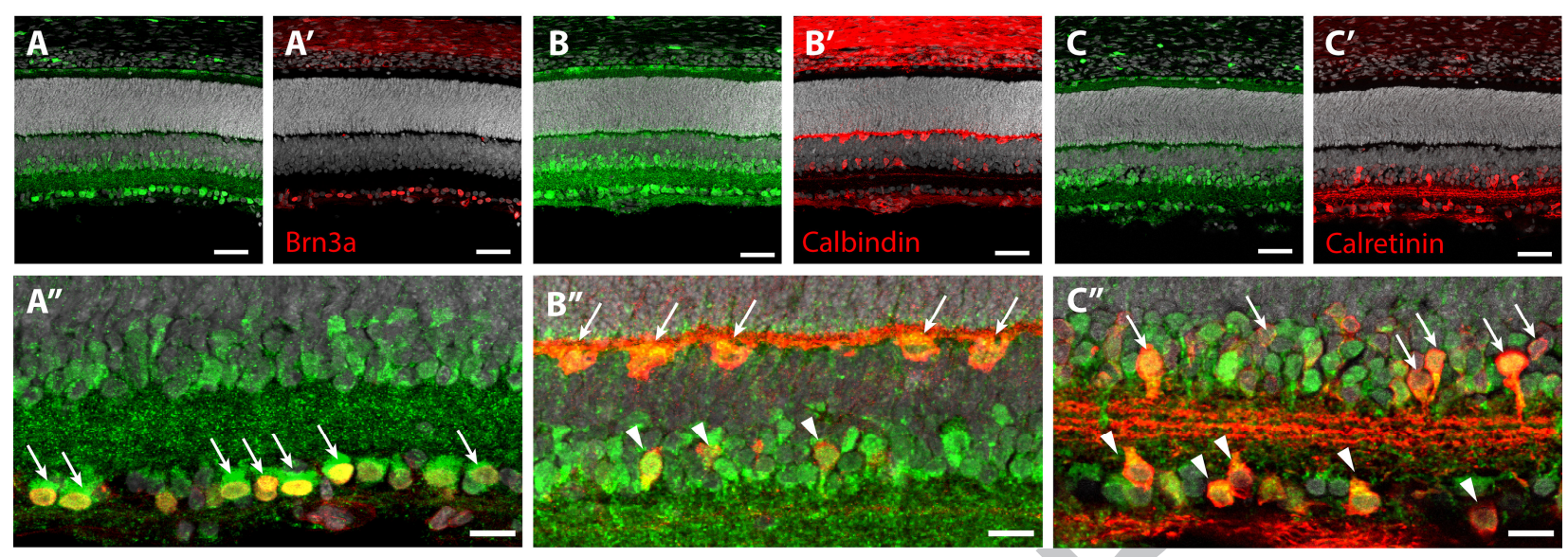
Figure 3
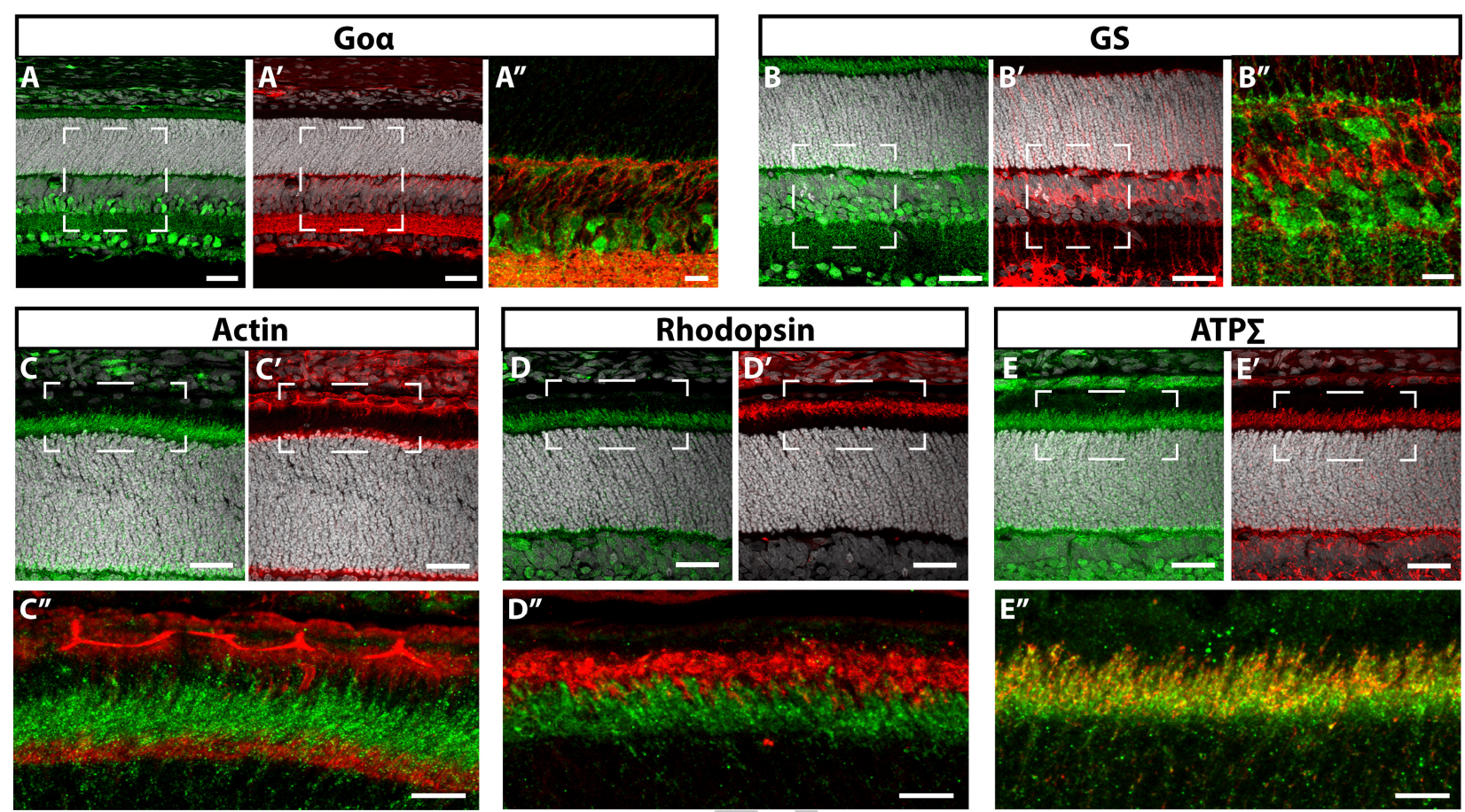
Figure 4
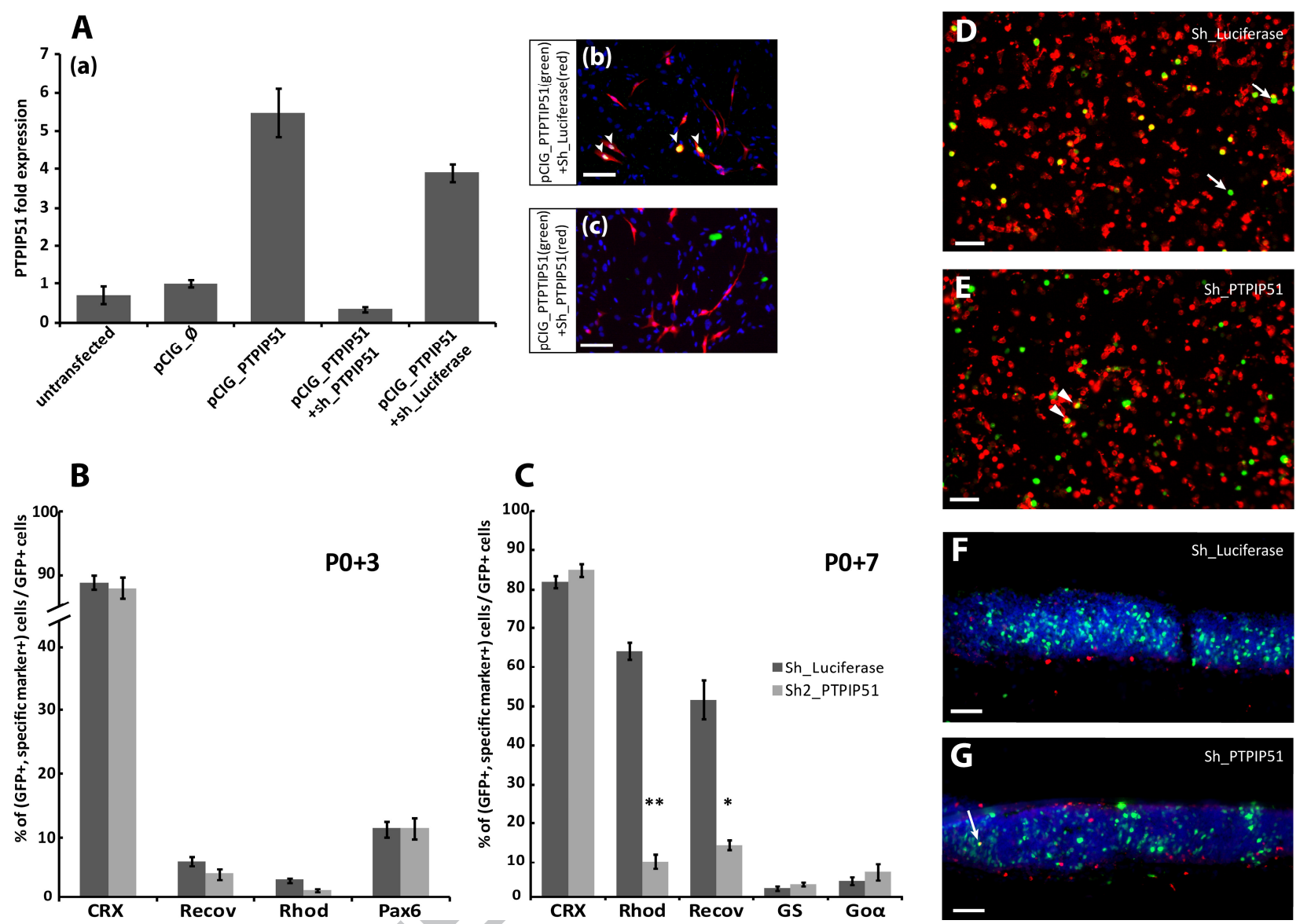
Figure 5

A

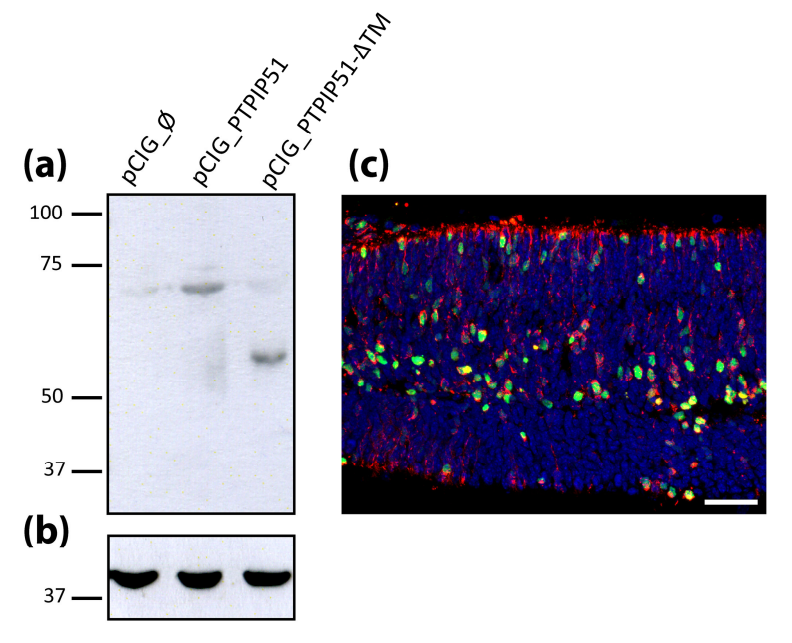

C

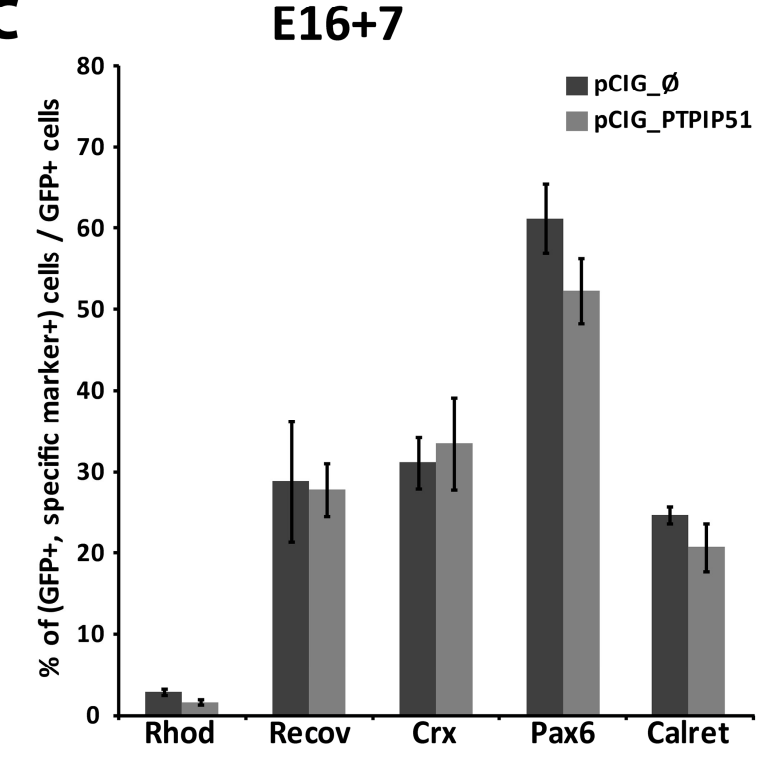

B

PO+9

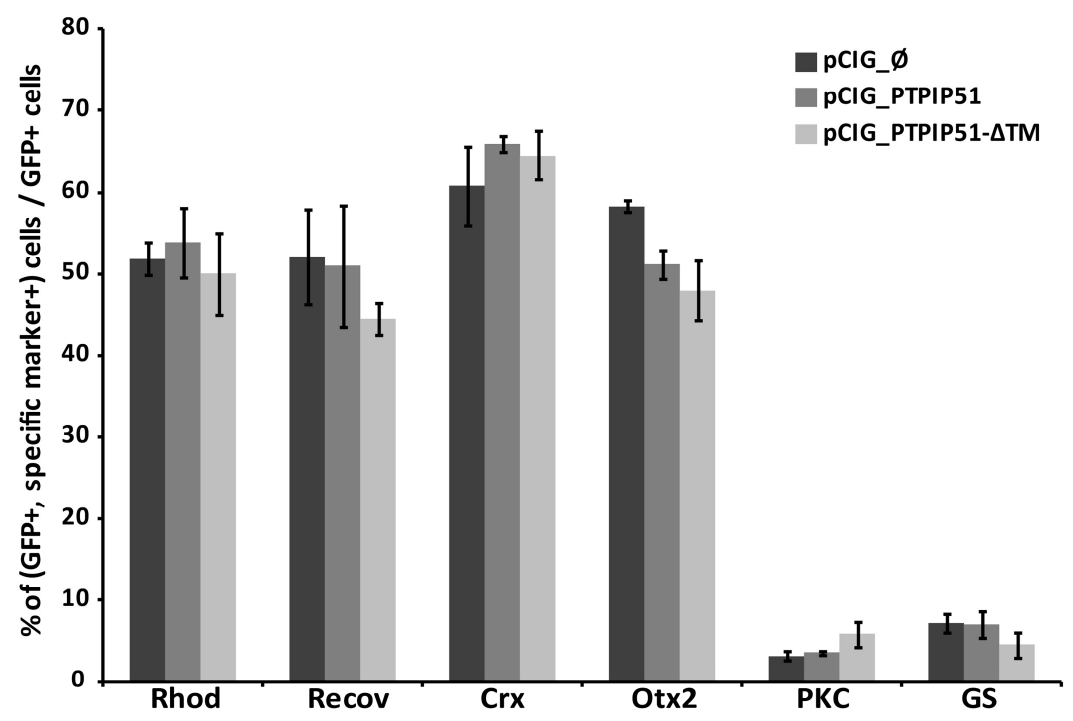

D
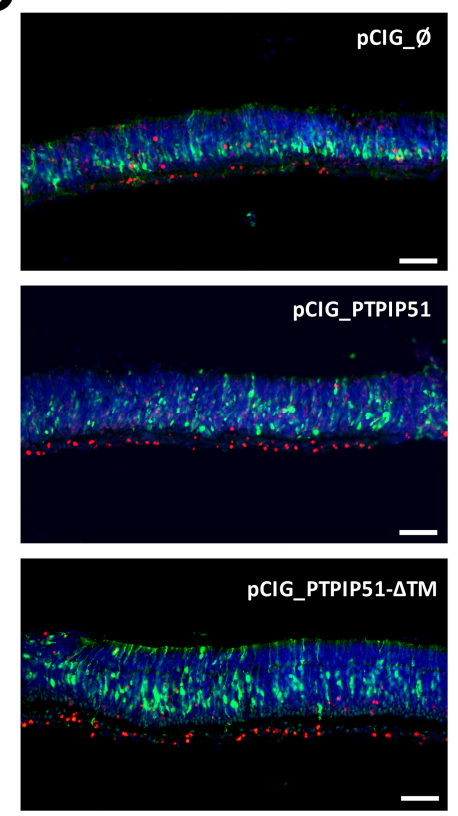